\title{
Air Activation Following an Atmospheric Explosion
}

\author{
JD Lowrey \\ JI Mclntyre \\ AW Prichard \\ CJ Gesh
}

October 2013

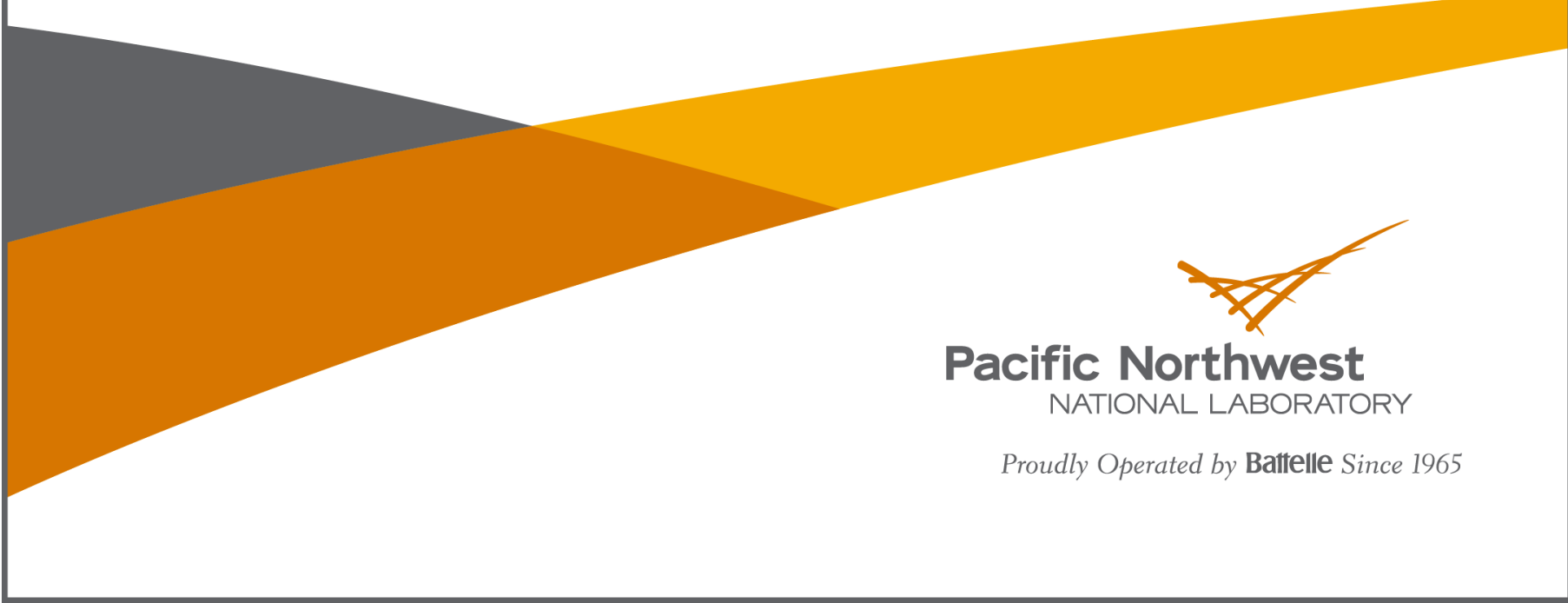




\section{DISCLAIMER}

This report was prepared as an account of work sponsored by an agency of the United States Government. Neither the United States Government nor any agency thereof, nor Battelle Memorial Institute, nor any of their employees, makes any warranty, express or implied, or assumes any legal liability or responsibility for the accuracy, completeness, or usefulness of any information, apparatus, product, or process disclosed, or represents that its use would not infringe privately owned rights. Reference herein to any specific commercial product, process, or service by trade name, trademark, manufacturer, or otherwise does not necessarily constitute or imply its endorsement, recommendation, or favoring by the United States Government or any agency thereof, or Battelle Memorial Institute. The views and opinions of authors expressed herein do not necessarily state or reflect those of the United States Government or any agency thereof.

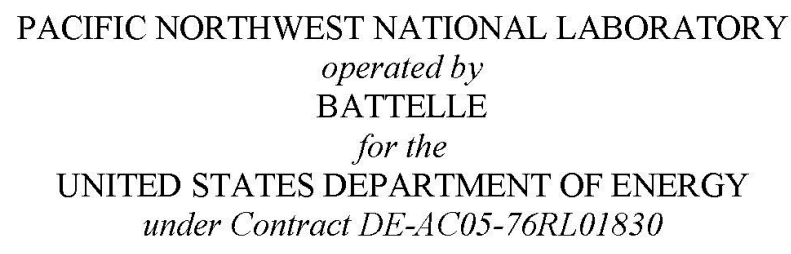

Printed in the United States of America

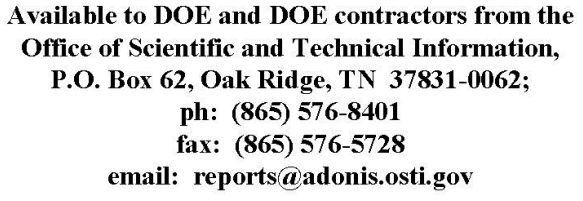

Available to the public from the National Technical Information Service

5301 Shawnee Rd., Alexandria, VA 22312 ph: (800) 553-NTIS (6847)

email: orders@ntis.gov <http://www.ntis.gov/about/form.aspx>

Online ordering: http://www.ntis.gov 


\title{
Air Activation Following an Atmospheric Explosion
}

\author{
JD Lowrey \\ JI Mclntyre \\ AW Prichard \\ CJ Gesh
}

October 2013

Prepared for

the U.S. Department of Energy

under Contract DE-AC05-76RL01830

Pacific Northwest National Laboratory

Richland, Washington 99352 



\section{Executive Summary}

In addition to thermal radiation and fission products, nuclear explosions result in a very high flux of neutrons. Within an atmospheric nuclear explosion, these neutrons can activate the various elemental components of natural air, potentially adding to the radioactive signature of the event as a whole. The goal of this work is to make an order-of-magnitude estimate of the total amount of air activation products that can result from an atmospheric nuclear explosion. 



\section{Contents}

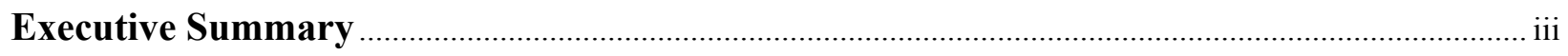

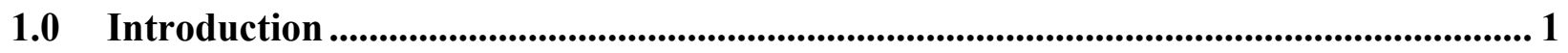

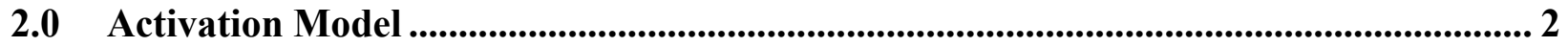

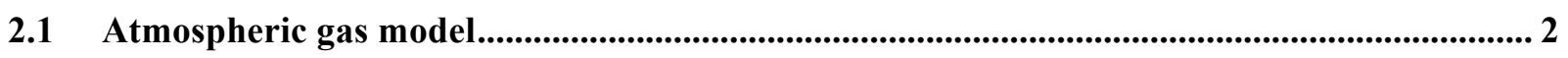

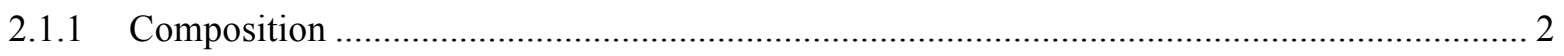

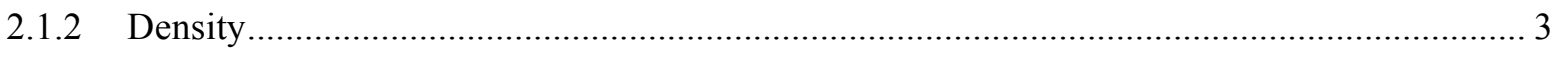

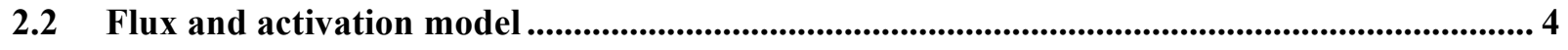

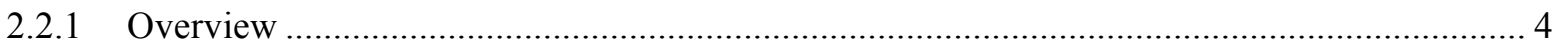

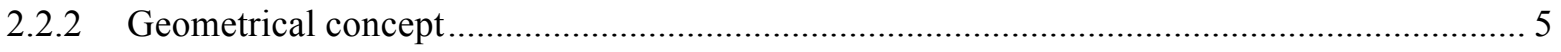

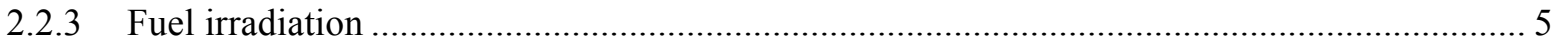

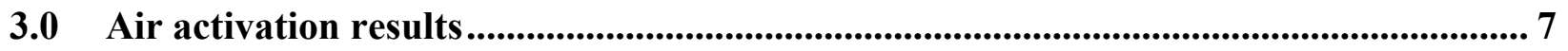

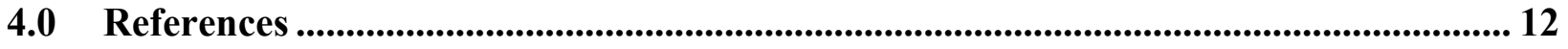




\section{Figures}

Figure 1: Atmospheric air density as a function altitude.

Figure 2: Air activation model geometry (not to scale) ........................................................................ 5

Figure 3: Estimated concentration of activated xenon as a function of radial distance........................... 7

Figure 4: Estimated concentration of activated argon as a function of radial distance .......................... 8

Figure 5: Estimated concentration of activated krypton as a function of radial distance ........................ 8

Figure 6: Integrated activated xenon as a function of radial distance in air ........................................ 9

\section{Tables}

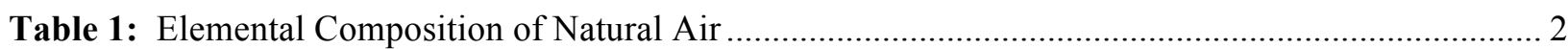

Table 2: Isotopic Fractions of Three Naturally-Occurring Noble Gases ............................................. 3

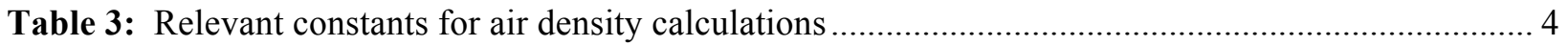

Table 4: Generic HEU and Plutonium Compositions (Stacey 2001) ................................................ 6

Table 5: Estimated total activities (in curies) of activation products in air at two average altitudes

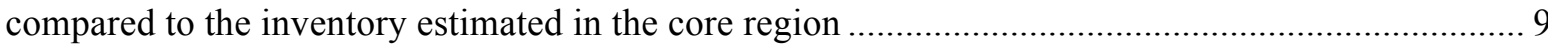




\subsection{Introduction}

A 1-kiloton-yield nuclear explosion requires around a mole of fission events releasing about $200 \mathrm{MeV}$ per fission. If the resulting neutron flux is only a function of the neutrons generated by fission events, and the average number of neutrons produced per fission is $v>2$, then the number of free neutrons released following the event is still on the order of $10^{23}$. Clearly even a small detonation results in a very high neutron flux. As these neutrons escape the exploding core, they will eventually be absorbed somewhere in the surrounding medium and the neutron flux will fall off as a function of distance due to both this absorption and geometric attenuation.

Neutrons moving through atmospheric air will activate nuclei, the net result of which is that the high neutron flux from an atmospheric nuclear explosion can potentially yield a great deal of activation products. Determining whether these activation products might be produced in great enough quantities to significantly alter the overall event isotopic signature is the goal of this study. To accomplish this, the neutron flux resulting from a 1-kiloton fission event was used to simulate activation of atmospheric air at multiple altitudes. The resulting amounts of activation products are then compared to the estimated fission products. 


\subsection{Activation Model}

To estimate the total inventory of air activation products that might result from an atmospheric nuclear explosion, an activation model is needed. To create this model, three major components are required:

1. A model of natural air characterized by elemental and isotopic composition, as well as altitudeadjusted density

2. A model for the neutron flux resulting from a hypothetical nuclear explosion

3. A model for simulation of activation of atmospheric air due to the estimated neutron flux

\subsection{Atmospheric gas model}

\subsubsection{Composition}

Table 1 shows the elemental composition of natural air assumed in this study (Köhne and Wößner 2009). For each element listed in Table 1, the natural isotopic abundances were determined; Table 2 lists isotopic fractions for the three nobles gases argon, krypton and xenon. Since the air composition determines the nuclei in the medium to be neutron-activated, this specification has a highly significant effect on the resulting activation product estimates, especially for ultra-trace gases such as xenon and argon.

Recognizing that air composition varies locally around the earth, the natural abundances assumed in this study represent a generalization.

Table 1: Elemental Composition of Natural Air

\begin{tabular}{ccc}
\hline $\mathbf{Z}$ & Element & ppm air \\
\hline 1 & $\mathrm{H}$ & 0.53 \\
2 & $\mathrm{He}$ & 5.2 \\
6 & $\mathrm{C}$ & 336.7 \\
7 & $\mathrm{~N}$ & 780900 \\
8 & $\mathrm{O}$ & 209500 \\
10 & $\mathrm{Ne}$ & 18 \\
16 & $\mathrm{~S}$ & 1 \\
18 & $\mathrm{Ar}$ & 9300 \\
36 & $\mathrm{Kr}$ & 1.14 \\
54 & $\mathrm{Xe}$ & 0.086 \\
\hline
\end{tabular}


PNNL-23023

Table 2: Isotopic Fractions of Three Naturally-Occurring Noble Gases

\begin{tabular}{cc|cc|cc}
\hline Isotope & Fraction & Isotope & Fraction & Isotope & Fraction \\
\hline${ }^{36} \mathrm{Ar}$ & $3.34 \times 10^{-3}$ & ${ }^{78} \mathrm{Kr}$ & $3.55 \times 10^{-03}$ & ${ }^{124} \mathrm{Xe}$ & $9.52 \times 10^{-4}$ \\
${ }^{38} \mathrm{Ar}$ & $6.29 \times 10^{-04}$ & ${ }^{80} \mathrm{Kr}$ & $2.29 \times 10^{-02}$ & ${ }^{126} \mathrm{Xe}$ & $8.9 \times 10^{-4}$ \\
${ }^{40} \mathrm{Ar}$ & .996 & ${ }^{82} \mathrm{Kr}$ & $1.16 \times 10^{-01}$ & ${ }^{128} \mathrm{Xe}$ & 0.0191 \\
& & ${ }^{83} \mathrm{Kr}$ & 0.115 & ${ }^{129} \mathrm{Xe}$ & 0.264 \\
& & ${ }^{84} \mathrm{Kr}$ & 0.570 & ${ }^{130} \mathrm{Xe}$ & 0.0407 \\
& ${ }^{86} \mathrm{Kr}$ & 0.173 & ${ }^{131} \mathrm{Xe}$ & 0.212 \\
& & & & ${ }^{132} \mathrm{Xe}$ & 0.269 \\
& & & & ${ }^{134} \mathrm{Xe}$ & 0.104 \\
& & & & & \\
& & & & & \\
\end{tabular}

\subsubsection{Density}

The density of air at sea level is about $1.225 \mathrm{~kg} / \mathrm{m}^{3}$. Since it is of interest to estimate activated air concentrations at various altitudes of detonation, the effect of altitude on air density must be accounted for. Air density can be calculated according to the ideal gas law by

$$
\rho=\frac{P M}{R T}
$$

where $M$ is the molar mass of air and $R$ is the ideal gas constant. Additionally, Equation (1) contains temperature $T$ and pressure $P$, which both depend on altitude. The variation of temperature with altitude $h$ is approximately linear and can be estimated by

$$
T(h)=T_{0}-L h,
$$

where $L$ is the temperature lapse rate. Air pressure as a function of altitude is given by

$$
P(h)=P_{0}\left(1-\frac{L h}{T_{0}}\right)^{\frac{g M}{R T}}
$$

where $g$ is the gravitational acceleration. Table 3 lists values for the constants appearing in Equations (1-3). Figure 1 shows a plot of air densities calculated from these equations. 
PNNL-23023

Table 3: Relevant constants for air density calculations

\begin{tabular}{ccc}
\hline atmospheric pressure (sea-level) & $\mathrm{P}_{0}$ & $101.325 \mathrm{kPa}$ \\
temperature (sea-level) & $\mathrm{T}_{0}$ & $288.15 \mathrm{~K}$ \\
gravitational acceleration (surface) & $\mathrm{g}$ & $9.80665 \mathrm{~m} / \mathrm{s}^{2}$ \\
temperature lapse rate & $\mathrm{L}$ & $0.0065 \mathrm{~K} / \mathrm{m}$ \\
ideal gas constant & $\mathrm{R}$ & $8.31447 \mathrm{~J} /(\mathrm{mol} \mathrm{K})$ \\
molar mass of dry air & $\mathrm{M}$ & $0.0289644 \mathrm{~kg} / \mathrm{mol}$ \\
\hline
\end{tabular}

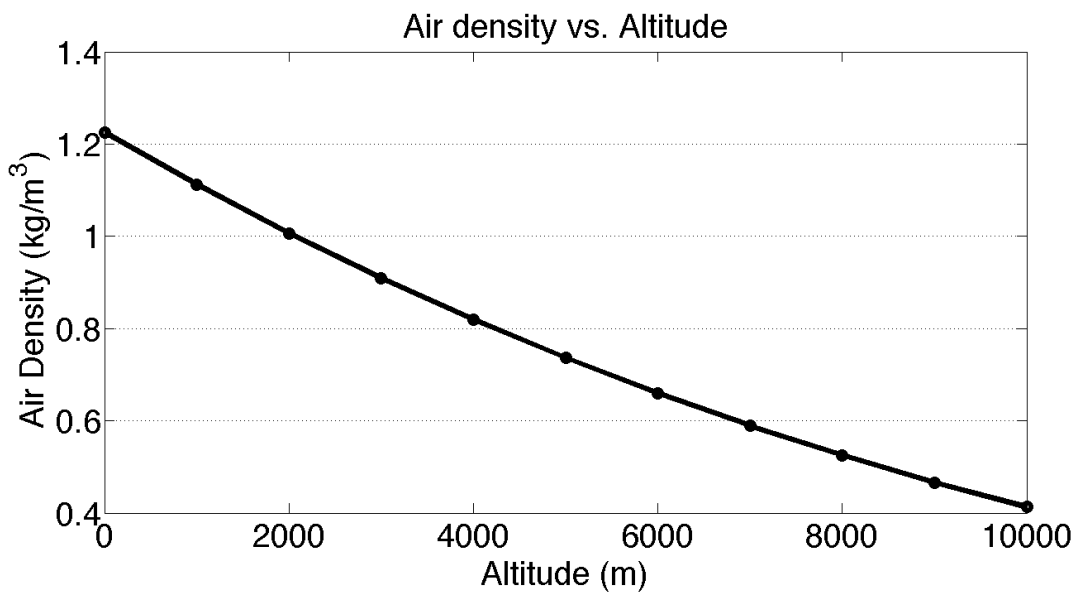

Figure 1: Atmospheric air density as a function altitude.

\subsection{Flux and activation model}

\subsubsection{Overview}

The air activation model, including the neutron flux estimation, was made using a burnup model consisting of

1. MCNP5: simulates the neutron flux resulting from a given specification of system geometry and nuclide composition

2. ORIGEN 2.2: estimates the fuel and air irradiation

3. MONTEBURNS: script that breaks a burnup event into discrete steps, coupling flux profiles from MCNP with irradiation in ORIGEN 2.2, adjusting system compositions and recalculating neutron fluxes with each step 
PNNL-23023

\subsubsection{Geometrical concept}

Flux and material estimates are estimated in discrete geometrical cells within MCNP to account for a nonuniform neutron flux throughout the system. The modeled system is depicted qualitatively in Figure 2. The fuel is modeled as a bare spherical reactor (see following section) surrounded by an extensive air medium formed by concentric shells of increasing thickness. For each cell in the geometric model, MCNP estimates a neutron flux profile within a uniform medium, which is fed into ORIGEN2.2 to estimate the resulting burnup and activation of the material in that cell at each time step.

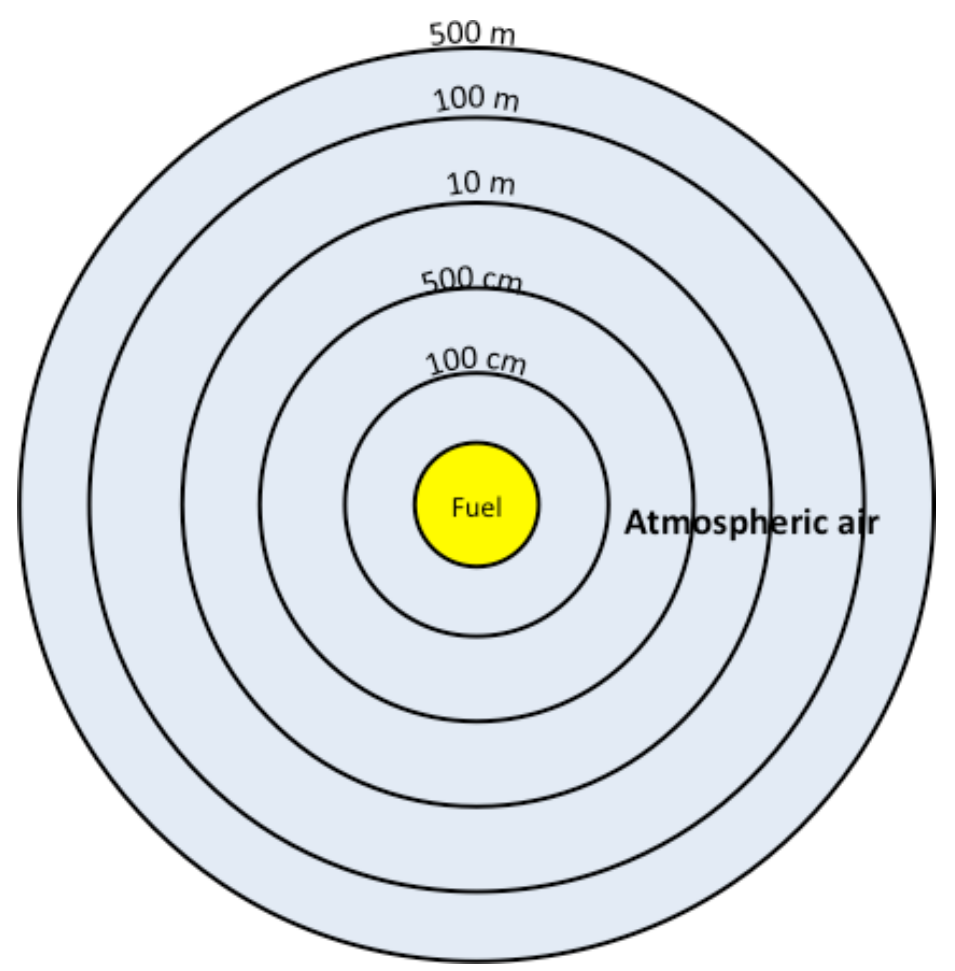

Figure 2: Air activation model geometry (not to scale)

\subsubsection{Fuel irradiation}

Two fuel types were considered in the activation calculations: highly enriched uranium (HEU) and plutonium, generic compositions of which are shown in Table 4 (Stacey 2001). Calculation of the fuel burnup was made assuming a Watts fission spectrum. To estimate the resulting neutron flux from a 1kiloton yield, burnup was modeled for a power of $4.18 \times 10^{12} \mathrm{MW}$ for a time of $1 \mu \mathrm{s}$, where $200 \mathrm{MeV}$ per fission gives about $1.3076 \times 10^{23}$ total fissions. 
PNNL-23023

Table 4: Generic HEU and Plutonium Compositions (Stacey 2001)

\begin{tabular}{cccc}
\hline \multicolumn{2}{c}{ HEU } & \multicolumn{2}{c}{$\mathrm{Pu}$} \\
\hline${ }^{234} \mathrm{U}$ & 0.12 & ${ }^{238} \mathrm{Pu}$ & 0.01 \\
${ }^{235} \mathrm{U}$ & 94.00 & ${ }^{239} \mathrm{Pu}$ & 93.80 \\
${ }^{238} \mathrm{U}$ & 5.88 & ${ }^{240} \mathrm{Pu}$ & 5.80 \\
& & ${ }^{241} \mathrm{Pu}$ & 0.13 \\
& & ${ }^{242} \mathrm{Pu}$ & 0.02 \\
& & ${ }^{241} \mathrm{Am}$ & 0.22 \\
\hline
\end{tabular}




\subsection{Air activation results}

Figures 3, 4 and 5 show plots of estimated activation product concentrations as a function of radial distance from the fuel region at sea level. Concentrations were estimated by dividing the total isotope inventory in each shell by the volume of that shell; the radius on the x-axis of these plots corresponds to the outer radius of the respective shell. With increasing radius, the neutron flux undergoes attenuation so that even though outer shells have a much higher volume of material, the concentration of activated nuclei falls off.

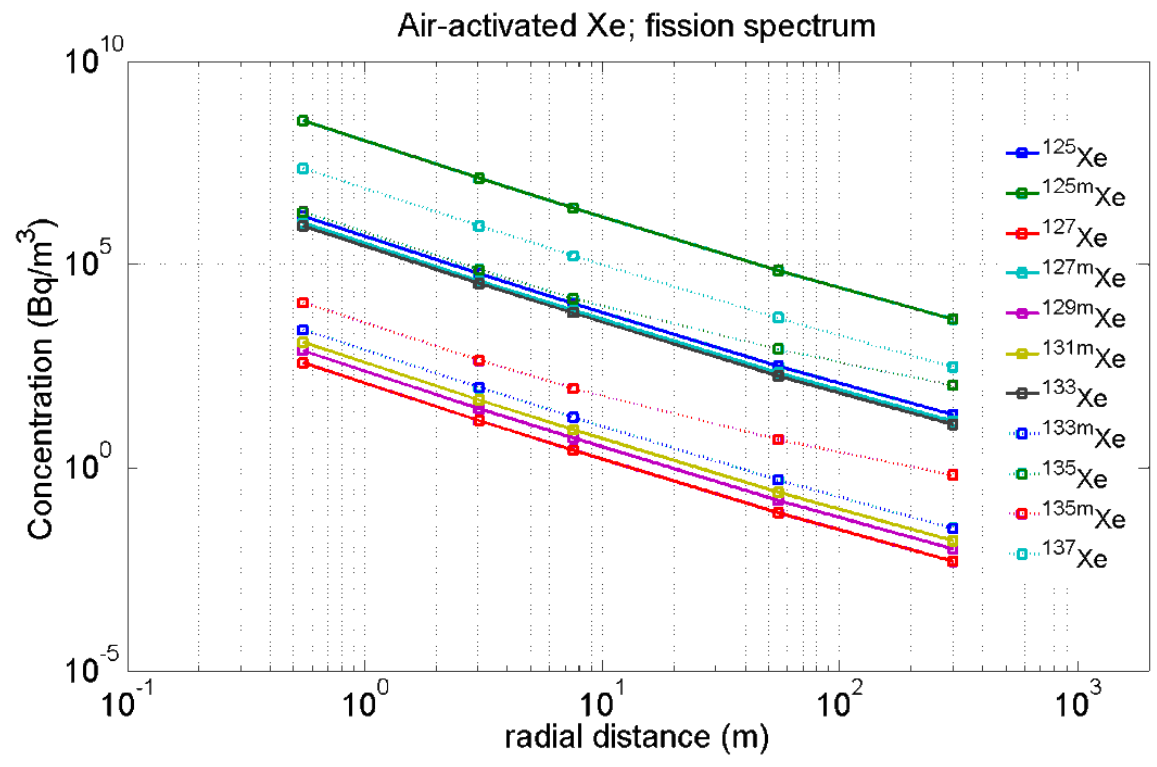

Figure 3: Estimated concentration of activated xenon as a function of radial distance 
PNNL-23023

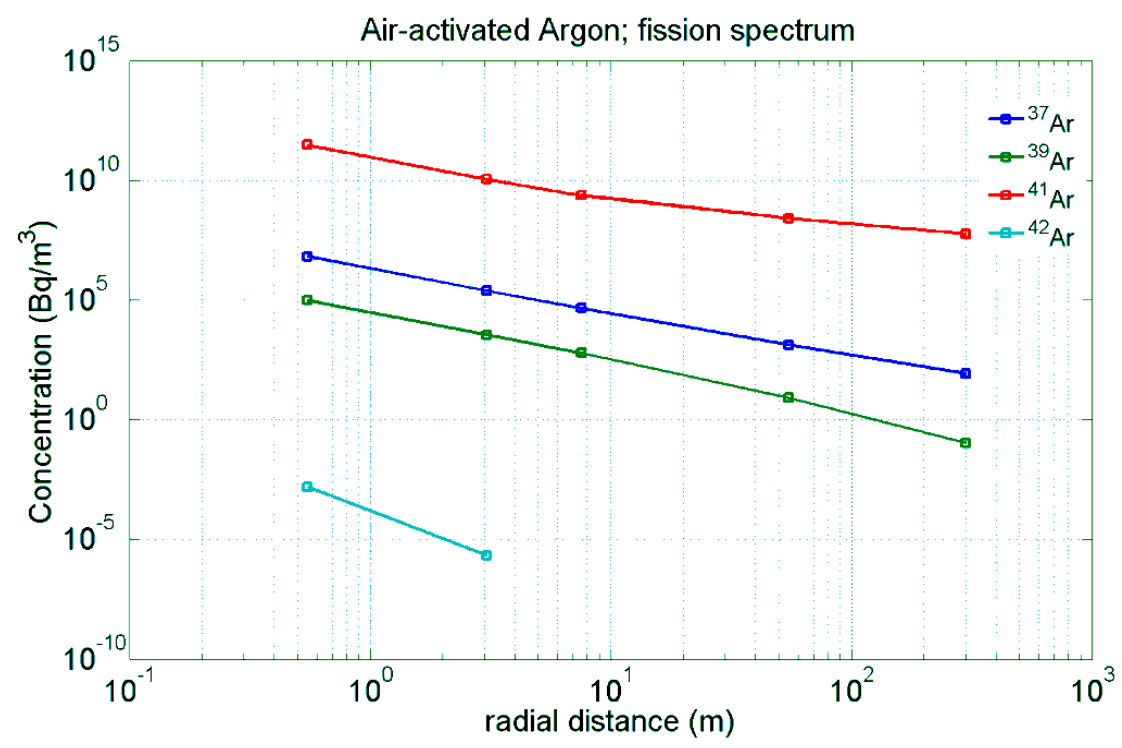

Figure 4: Estimated concentration of activated argon as a function of radial distance

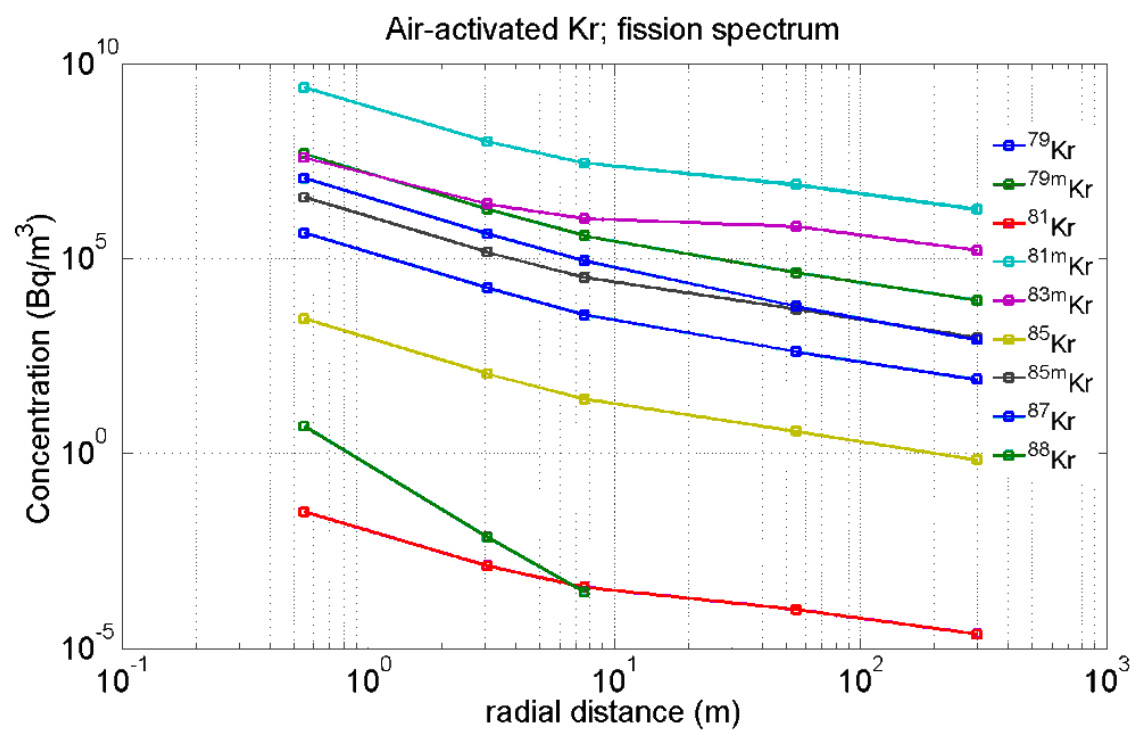

Figure 5: Estimated concentration of activated krypton as a function of radial distance

Figure 6 shows a plot of the integrated activated xenon activity as function of radial distance from the fuel region. At roughly $1 \mathrm{~km}$ radial distance, the neutron flux has decreased to the point that further activated xenon contributes negligibly to the total inventory. As such, air activation from a 1-kiloton atmospheric nuclear explosion has effectively ceased beyond $1 \mathrm{~km}$ radius. 
Table 5 summarizes total activities (in curies) integrated over all simulated air regions of activation products in air at two average altitudes. Also shown for comparison are the estimated inventories due to fission in the respective cores.

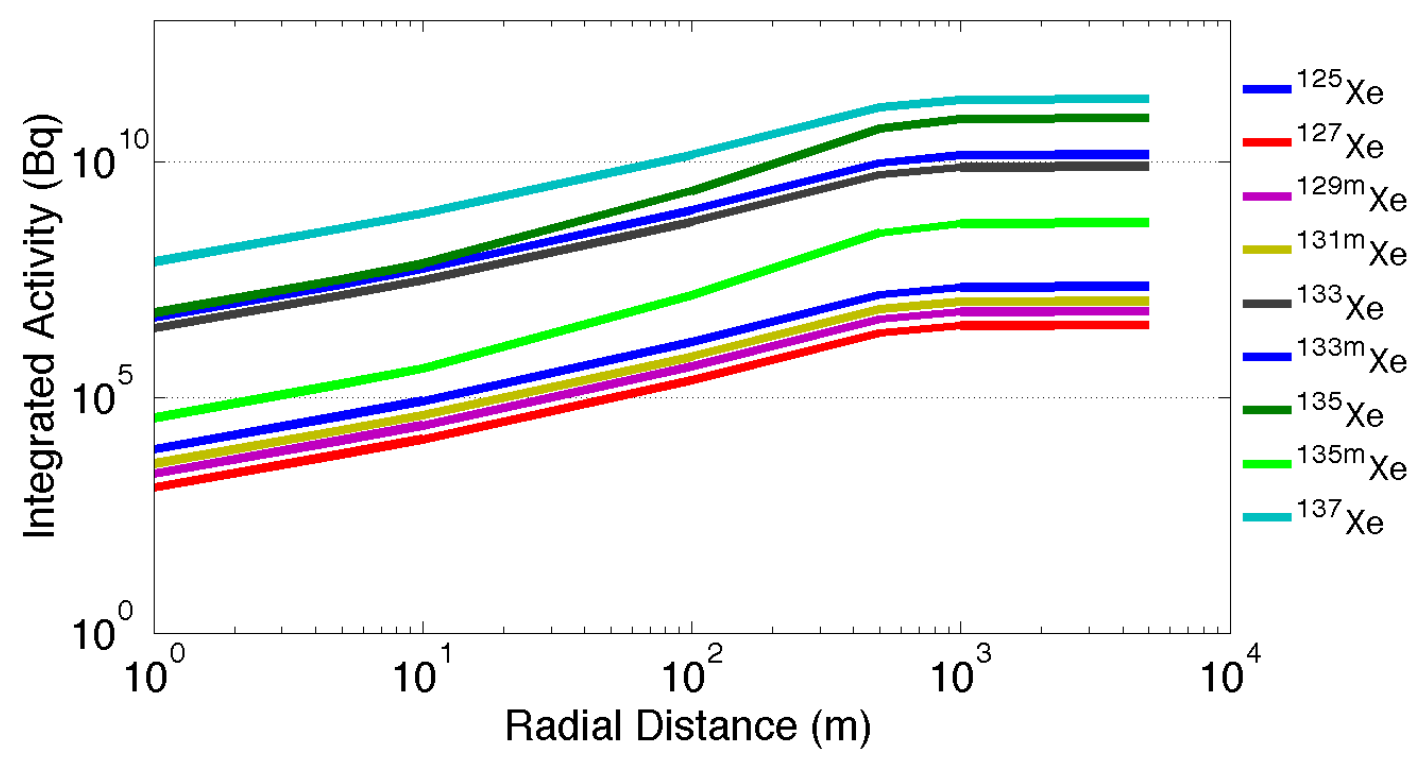

Figure 6: Integrated activated xenon as a function of radial distance in air

Table 5: Estimated total activities (in curies) of activation products in air at two average altitudes compared to the inventory estimated in the core region

\begin{tabular}{l|ccc|ccc}
\hline \multicolumn{2}{l}{} & & Pu & & \multicolumn{3}{c}{ HEU } \\
\multicolumn{1}{c}{ Core } & $\mathbf{2 ~ k m ~ e l e v . ~}$ & $\mathbf{1 0} \mathbf{~ k m ~ e l e v . ~}$ & Core & $\mathbf{2 ~ k m ~ e l e v . ~}$ & $\mathbf{1 0 ~ k m ~ e l e v . ~}$ \\
\hline${ }^{6} \mathrm{He}$ & - & $3.57 \times 10^{-04}$ & $8.49 \times 10^{-05}$ & - & $8.25 \times 10^{-05}$ & $3.37 \times 10^{-05}$ \\
${ }^{8} \mathrm{Li}$ & - & $5.64 \times 10^{-01}$ & $1.38 \times 10^{-01}$ & - & $9.38 \times 10^{-02}$ & $3.70 \times 10^{-02}$ \\
${ }^{8} \mathrm{Be}$ & - & $4.43 \times 10^{02}$ & $1.06 \times 10^{02}$ & - & $9.87 \times 10^{01}$ & $4.01 \times 10^{01}$ \\
${ }^{10} \mathrm{Be}$ & - & $2.21 \times 10^{-09}$ & $2.24 \times 10^{-09}$ & - & $1.63 \times 10^{-09}$ & $1.65 \times 10^{-09}$ \\
${ }^{11} \mathrm{Be}$ & - & $7.56 \times 10^{-04}$ & $1.83 \times 10^{-04}$ & - & $1.25 \times 10^{-04}$ & $4.10 \times 10^{-05}$ \\
${ }^{12} \mathrm{~B}$ & - & $6.07 \times 10^{07}$ & $6.36 \times 10^{07}$ & - & $3.27 \times 10^{07}$ & $2.97 \times 10^{07}$ \\
${ }^{14} \mathrm{C}$ & $1.69 \times 10^{-07}$ & $2.31 \times 10^{01}$ & $2.34 \times 10^{01}$ & $1.75 \times 10^{-07}$ & $1.75 \times 10^{01}$ & $1.77 \times 10^{01}$ \\
${ }^{15} \mathrm{C}$ & - & $9.74 \times 10^{04}$ & $1.05 \times 10^{05}$ & - & $5.35 \times 10^{04}$ & $4.64 \times 10^{04}$ \\
${ }^{13} \mathrm{~N}$ & - & $1.23 \times 10^{04}$ & $1.24 \times 10^{04}$ & - & $6.54 \times 10^{03}$ & $4.70 \times 10^{03}$ \\
${ }^{16} \mathrm{~N}$ & - & $5.10 \times 10^{06}$ & $5.49 \times 10^{06}$ & - & $2.77 \times 10^{06}$ & $2.38 \times 10^{06}$ \\
${ }^{29} \mathrm{Al}$ & - & $6.71 \times 10^{-09}$ & $1.38 \times 10^{-09}$ & - & $1.32 \times 10^{-09}$ & $5.43 \times 10^{-10}$ \\
${ }^{31} \mathrm{Si}$ & - & $1.42 \times 10^{-01}$ & $1.44 \times 10^{-01}$ & - & $1.05 \times 10^{-01}$ & $1.06 \times 10^{-01}$ \\
${ }^{32} \mathrm{P}$ & - & $4.08 \times 10^{-01}$ & $4.17 \times 10^{-01}$ & - & $2.29 \times 10^{-01}$ & $2.32 \times 10^{-01}$ \\
${ }^{33} \mathrm{P}$ & - & $2.97 \times 10^{-07}$ & $3.00 \times 10^{-07}$ & - & $2.19 \times 10^{-07}$ & $2.22 \times 10^{-07}$ \\
${ }^{34} \mathrm{P}$ & - & $2.72 \times 10^{01}$ & $2.76 \times 10^{01}$ & - & $2.01 \times 10^{01}$ & $2.04 \times 10^{01}$ \\
${ }^{35} \mathrm{~S}$ & - & $5.71 \times 10^{-05}$ & $5.78 \times 10^{-05}$ & - & $4.22 \times 10^{-05}$ & $4.27 \times 10^{-05}$ \\
${ }^{37} \mathrm{~S}$ & - & $4.54 \times 10^{04}$ & $4.66 \times 10^{04}$ & - & $2.44 \times 10^{04}$ & $2.41 \times 10^{04}$ \\
\hline
\end{tabular}


PNNL-23023

\begin{tabular}{|c|c|c|c|c|c|c|}
\hline & \multicolumn{3}{|c|}{$\mathrm{Pu}$} & \multicolumn{3}{|c|}{ HEU } \\
\hline & Core & $2 \mathrm{~km}$ elev. & 10 km elev. & Core & 2 km elev. & $10 \mathrm{~km}$ elev. \\
\hline${ }^{36} \mathrm{Cl}$ & - & $2.91 \times 10^{-06}$ & $2.95 \times 10^{-06}$ & - & $2.15 \times 10^{-06}$ & $2.18 \times 10^{-06}$ \\
\hline${ }^{37} \mathrm{Ar}$ & - & $2.40 \times 10^{00}$ & $2.43 \times 10^{00}$ & - & $1.77 \times 10^{00}$ & $1.79 \times 10^{00}$ \\
\hline${ }^{39} \mathrm{Ar}$ & - & $2.76 \times 10^{-03}$ & $2.92 \times 10^{-03}$ & - & $1.51 \times 10^{-03}$ & $1.26 \times 10^{-03}$ \\
\hline${ }^{41} \mathrm{Ar}$ & - & $2.37 \times 10^{06}$ & $2.40 \times 10^{06}$ & - & $1.83 \times 10^{06}$ & $1.85 \times 10^{06}$ \\
\hline${ }^{42} \mathrm{~A}$ & - & $3.26 \times 10^{-13}$ & $7.61 \times 10^{-14}$ & - & $1.00 \times 10^{-13}$ & $4.09 \times 10^{-14}$ \\
\hline${ }^{42} \mathrm{~K}$ & - & - & - & - & - & - \\
\hline${ }^{75} \mathrm{~S}$ & - & $5.57 \times 10^{-06}$ & $5.70 \times 10^{-06}$ & - & - & - \\
\hline${ }^{79} \mathrm{Se}$ & $9.94 \times 10^{-07}$ & $7.85 \times 10^{-13}$ & $3.60 \times 10^{-13}$ & $8.96 \times 10^{-08}$ & $4.86 \times 10^{-14}$ & $1.91 \times 10^{-13}$ \\
\hline${ }^{81} \mathrm{Se}$ & $1.17 \times 10^{05}$ & $2.27 \times 10^{-03}$ & $2.31 \times 10^{-03}$ & $4.71 \times 10^{04}$ & $1.19 \times 10^{-03}$ & $1.14 \times 10^{-03}$ \\
\hline${ }^{80} \mathrm{Br}$ & $3.89 \times 10^{01}$ & $2.99 \times 10^{-01}$ & $3.06 \times 10^{-01}$ & $3.27 \times 10^{00}$ & $1.64 \times 10^{-01}$ & $1.66 \times 10^{-01}$ \\
\hline${ }^{82} \mathrm{Br}$ & $1.22 \times 10^{02}$ & $1.42 \times 10^{-03}$ & $1.46 \times 10^{-03}$ & $2.12 \times 10^{01}$ & $7.75 \times 10^{-04}$ & $7.76 \times 10^{-04}$ \\
\hline${ }^{83} \mathrm{Br}$ & $3.30 \times 10^{04}$ & $1.57 \times 10^{-02}$ & $1.60 \times 10^{-02}$ & $8.38 \times 10^{03}$ & $8.67 \times 10^{-03}$ & $8.76 \times 10^{-03}$ \\
\hline${ }^{79} \mathrm{Kr}$ & - & $2.57 \times 10^{00}$ & $2.60 \times 10^{00}$ & - & $1.14 \times 10^{-02}$ & $1.15 \times 10^{-02}$ \\
\hline${ }^{79 m} \mathrm{Kr}$ & - & $2.73 \times 10^{02}$ & $2.77 \times 10^{02}$ & - & $1.21 \times 10^{00}$ & $1.22 \times 10^{00}$ \\
\hline${ }^{81} \mathrm{Kr}$ & - & $7.80 \times 10^{-07}$ & $7.90 \times 10^{-07}$ & - & $6.04 \times 10^{-07}$ & $6.11 \times 10^{-07}$ \\
\hline${ }^{81 m_{K r}}$ & $6.53 \times 10^{01}$ & $6.16 \times 10^{04}$ & $6.24 \times 10^{04}$ & - & $4.78 \times 10^{04}$ & $4.83 \times 10^{04}$ \\
\hline${ }^{83 m_{K r}}$ & $1.75 \times 10^{02}$ & $5.74 \times 10^{03}$ & $5.83 \times 10^{03}$ & $9.94 \times 10^{00}$ & $4.46 \times 10^{03}$ & $4.49 \times 10^{03}$ \\
\hline${ }^{85} \mathrm{Kr}$ & $5.47 \times 10^{-01}$ & $2.12 \times 10^{-02}$ & $2.12 \times 10^{-02}$ & $1.42 \times 10^{-01}$ & $1.63 \times 10^{-02}$ & $1.64 \times 10^{-02}$ \\
\hline${ }^{85 m_{1}} \mathrm{Kr}$ & $1.34 \times 10^{04}$ & $2.87 \times 10^{01}$ & $2.87 \times 10^{01}$ & $5.26 \times 10^{03}$ & $2.20 \times 10^{01}$ & $2.21 \times 10^{01}$ \\
\hline${ }^{87} \mathrm{Kr}$ & $1.74 \times 10^{06}$ & $2.51 \times 10^{01}$ & $2.55 \times 10^{01}$ & $1.67 \times 10^{06}$ & $1.93 \times 10^{01}$ & $1.96 \times 10^{01}$ \\
\hline${ }^{88} \mathrm{Kr}$ & $1.96 \times 10^{06}$ & $1.08 \times 10^{-09}$ & $2.54 \times 10^{-10}$ & $2.91 \times 10^{06}$ & $3.38 \times 10^{-10}$ & $1.31 \times 10^{-10}$ \\
\hline${ }^{88} \mathrm{Rb}$ & $1.55 \times 10^{06}$ & - & - & $6.77 \times 10^{05}$ & - & - \\
\hline${ }^{127} \mathrm{Te}$ & $3.19 \times 10^{02}$ & $1.05 \times 10^{-06}$ & $1.07 \times 10^{-06}$ & $4.29 \times 10^{02}$ & $7.79 \times 10^{-07}$ & $7.87 \times 10^{-07}$ \\
\hline${ }^{129} \mathrm{Te}$ & $1.61 \times 10^{05}$ & $1.86 \times 10^{-05}$ & $1.88 \times 10^{-05}$ & $3.36 \times 10^{05}$ & $1.37 \times 10^{-05}$ & $1.39 \times 10^{-05}$ \\
\hline${ }^{125} I$ & - & $2.51 \times 10^{-14}$ & $2.54 \times 10^{-14}$ & - & $1.85 \times 10^{-14}$ & $1.88 \times 10^{-14}$ \\
\hline${ }^{128} I$ & $3.43 \times 10^{02}$ & $5.11 \times 10^{-05}$ & $5.17 \times 10^{-05}$ & $6.14 \times 10^{01}$ & $3.78 \times 10^{-05}$ & $3.82 \times 10^{-05}$ \\
\hline${ }^{130}{ }^{\prime}$ & $9.01 \times 10^{02}$ & $8.95 \times 10^{-06}$ & $9.06 \times 10^{-06}$ & $1.20 \times 10^{02}$ & $6.61 \times 10^{-06}$ & $6.69 \times 10^{-06}$ \\
\hline${ }^{131}$ I & $3.78 \times 10^{02}$ & $2.97 \times 10^{-06}$ & $3.04 \times 10^{-06}$ & $3.40 \times 10^{02}$ & $1.70 \times 10^{-06}$ & $1.67 \times 10^{-06}$ \\
\hline${ }^{132}$ & $3.46 \times 10^{05}$ & $8.27 \times 10^{-05}$ & $8.38 \times 10^{-05}$ & $1.85 \times 10^{05}$ & $6.11 \times 10^{-05}$ & $6.18 \times 10^{-05}$ \\
\hline & - & $3.74 \times 10^{-01}$ & $3.78 \times 10^{-01}$ & - & $2.76 \times 10^{-01}$ & $2.79 \times 10^{-01}$ \\
\hline & - & $8.33 \times 10^{01}$ & $8.43 \times 10^{01}$ & - & $6.16 \times 10^{01}$ & $6.22 \times 10^{01}$ \\
\hline${ }^{127} X$ & - & $9.15 \times 10^{-05}$ & $9.27 \times 10^{-05}$ & - & $6.77 \times 10^{-05}$ & $6.84 \times 10^{-05}$ \\
\hline & - & $2.53 \times 10^{-01}$ & $2.56 \times 10^{-01}$ & - & $1.87 \times 10^{-01}$ & $1.89 \times 10^{-01}$ \\
\hline & $9.03 \times 10^{-04}$ & $1.80 \times 10^{-04}$ & $1.82 \times 10^{-04}$ & $4.91 \times 10^{-04}$ & $1.33 \times 10^{-04}$ & $1.34 \times 10^{-04}$ \\
\hline 131 & $1.81 \times 10^{-01}$ & $2.94 \times 10^{-04}$ & $2.97 \times 10^{-04}$ & $1.35 \times 10^{-01}$ & $2.17 \times 10^{-04}$ & $2.19 \times 10^{-04}$ \\
\hline${ }^{133} \mathrm{Xe}$ & $6.99 \times 10^{02}$ & $2.10 \times 10^{-01}$ & $2.13 \times 10^{-01}$ & $1.95 \times 10^{02}$ & $1.55 \times 10^{-01}$ & $1.57 \times 10^{-01}$ \\
\hline & $5.44 \times 10^{03}$ & $5.95 \times 10^{-04}$ & $6.03 \times 10^{-04}$ & $1.55 \times 10^{03}$ & $4.40 \times 10^{-04}$ & $4.45 \times 10^{-04}$ \\
\hline${ }^{135} \mathrm{Xe}$ & $2.65 \times 10^{05}$ & $2.22 \times 10^{00}$ & $2.24 \times 10^{00}$ & $1.10 \times 10^{05}$ & $1.70 \times 10^{00}$ & $1.72 \times 10^{00}$ \\
\hline & $1.31 \times 10^{07}$ & $1.36 \times 10^{-02}$ & $1.37 \times 10^{-02}$ & $6.56 \times 10^{06}$ & $1.04 \times 10^{-02}$ & $1.05 \times 10^{-02}$ \\
\hline${ }^{137} \mathrm{Xe}$ & $3.61 \times 10^{08}$ & $5.63 \times 10^{00}$ & $5.70 \times 10^{00}$ & $2.84 \times 10^{08}$ & $4.16 \times 10^{00}$ & $4.20 \times 10^{00}$ \\
\hline${ }^{135} \mathrm{Cs}$ & $8.66 \times 10^{-07}$ & $1.06 \times 10^{-20}$ & $1.07 \times 10^{-20}$ & $8.89 \times 10^{-07}$ & $8.13 \times 10^{-21}$ & $8.21 \times 10^{-21}$ \\
\hline
\end{tabular}


PNNL-23023

\begin{tabular}{|c|c|c|c|c|c|c|}
\hline & \multicolumn{3}{|c|}{$\mathrm{Pu}$} & \multicolumn{3}{|c|}{ HEU } \\
\hline & Core & 2 km elev. & $10 \mathrm{~km}$ elev. & Core & $2 \mathrm{~km}$ elev. & $10 \mathrm{~km}$ elev. \\
\hline${ }^{137} \mathrm{Cs}$ & $1.46 \times 10^{01}$ & $2.06 \times 10^{-15}$ & $2.08 \times 10^{-15}$ & $6.48 \times 10^{00}$ & $1.52 \times 10^{-15}$ & $1.54 \times 10^{-15}$ \\
\hline${ }^{137 m_{B a}}$ & $1.66 \times 10^{05}$ & - & - & $7.38 \times 10^{04}$ & - & - \\
\hline Total & $7.92 \times 10^{11}$ & $6.84 \times 10^{07}$ & $7.17 \times 10^{07}$ & $1.41 \times 10^{12}$ & $3.74 \times 10^{07}$ & $3.40 \times 10^{07}$ \\
\hline
\end{tabular}


PNNL-23023

\subsection{References}

Köhne, A, and M Wößner. 2009. Earth's Atmosphere. Accessed on 2 October, 2013 at http://www.kowoma.de/en/gps/additional/atmosphere.htm (last updated 19 April 2009).

Stacey, WM. 2001. Nuclear Reactor Physics. First ed., John Wiley \& Sons, Inc., New York, NY. 


\section{Distribution}

No. of

Copies

\# Name

Organization

Address

City, State and ZIP Code

\# Organization

Address

City, State and ZIP Code

Name

Name

Name

Name

Name (\#)

\# Name

Organization

Address

City, State and ZIP Code
No. of

\section{Copies}

(1)




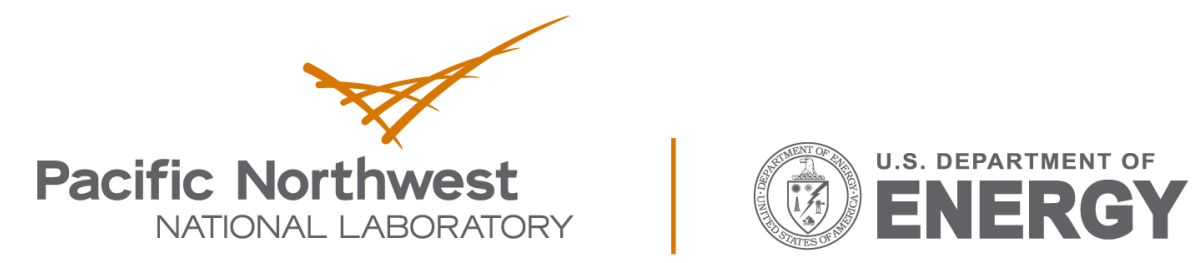

Proudly Operated by Battelle Since 1965

902 Battelle Boulevard

P.O. Box 999

Richland, WA 99352

1-888-375-PNNL (7665)

www.pnnl.gov 